\title{
Acurácia de dois dendrômetros ópticos na cubagem não destrutiva para a determinação da biomassa florestal
}

\author{
Marcos Felipe Nicoletti', João Luis Ferreira Batista², Samuel de Pádua Chaves Carvalho², Tito Nunes de Castro²
}

'Universidade do Estado de Santa Catarina, CEP 88.520-000, Lages, SC, Brasil.

${ }^{2}$ Universidade de São Paulo, CEP 13418-900, Piracicaba, SP, Brasil

"Autor correspondente:

a2mfn@cav.udesc.br

Termos para indexação:

Quantificação de biomassa

Criterion

$\mathrm{RC} 3 \mathrm{H}$ da Masser

Modelos empíricos

Index terms:

Biomass

Criterion

Masser RCH3

Empirical models

Histórico do artigo:

Recebido em 20/10/2011

Aprovado em 23/05/2012

Publicado em 29/06/2012

doi: $10.4336 / 2012 . p f b .32 .70 .23$
Resumo - O objetivo deste trabalho foi aprimorar os métodos existentes para determinação da biomassa do tronco sob forma não destrutiva, e verificar a influência que a medição de variáveis por meio de dendrômetros a laser exerce sobre a predição pelos modelos empíricos existentes no meio florestal, para árvores individuais. $\mathrm{O}$ estudo foi desenvolvido em Itatinga, SP, em três parcelas de um plantio de Eucalyptus grandis, totalizando 175 árvores. Os diâmetros ao longo do fuste das árvores em pé foram mensurados com dois dendrômetros, Criterion e $\mathrm{RC} 3 \mathrm{H}$, até os $8 \mathrm{~m}$ de altura. Após a cubagem em pé, as árvores foram derrubadas e obtidos os diâmetros nas mesmas posições em que se realizou a cubagem não destrutiva. Discos de madeira foram retirados e secos para determinação da umidade. A determinação da biomassa foi realizada através da técnica gravimétrica e da volumétrica por meio de três métodos diferentes (cubagem rigorosa tradicional, Criterion e $\mathrm{RC} 3 \mathrm{H}$ ). Em seguida ajustaram-se alguns modelos para as técnicas e selecionaram-se os de melhor ajuste. De modo geral, todos os modelos apresentaram bons ajustes. Os erros padrão da predição médios para estes modelos foram superestimados em $1 \%$ com a cubagem rigorosa tradicional e subestimados em aproximadamente $11 \%$ para o Criterion e $40 \%$ para o $\mathrm{RC} 3 \mathrm{H}$.

\section{Accuracy of two optical dendrometers for non destructive determination of woody biomass}

\begin{abstract}
The objective of this study was to improve existing methods for determination of stem biomass in a non-destructive way, and verify the influence that laser dendrometers have on the prediction by existing empirical models in forest environment on the individual trees. This study was developed in Itatinga, SP, Brazil, in three plots of a Eucalyptus grandis plantation, totalizing 175 trees. The stem diameter of standing trees were measured with two dendrometers, Criterion and $\mathrm{RC} 3 \mathrm{H}$, along the stem up to 8 meters height. After the volume evaluation of standing trees they were felled and the diameter obtained with a caliper and a tape in the same positions in which the non-destructive measures were taken. Wood disks were removed and dried for determination of humidity. The determination of biomass was performed by volumetric and gravimetric technique using three different methods (traditional wood volume evaluation, Criterion and RC3H). Some models were tested for these techniques and the best adjustment was selected. In general, all models showed good adjustments. The standard error average of prediction for these models were overestimated in $1 \%$ with the traditional wood volume evaluation and underestimated in approximately $11 \%$ using the Criterion and $40 \%$ using the $\mathrm{RC} 3 \mathrm{H}$.
\end{abstract}




\section{Introdução}

Devido à importância das florestas no cenário das mudanças climáticas, tem aumentado a demanda por pesquisas que quantifiquem o potencial dos ecossistemas florestais em sequestrar carbono da atmosfera (Melo \& Durigan, 2006). Para efeito de clima é extremamente importante que se realizem as estimativas de biomassa florestal, por estarem diretamente relacionadas com os estoques de carbono que, por sua vez, são utilizados para quantificar o gás carbônico liberado na atmosfera durante o processo de degradação florestal (Higuchi et al., 1998).

Atualmente, tem-se utilizado, também, a produção de biomassa, já que o conhecimento desta nos diversos ecossistemas tem assumido papel importante para um vasto conjunto de aspectos como comercialização de produtos, estudos da produtividade do sistema florestal, de fluxos de energia e de nutrientes e da contribuição dos ecossistemas para o ciclo global do carbono (Soares et al., 2005). Desta forma, quanto maior a precisão da estimativa de biomassa, maior será a consistência da quantificação de carbono estocado e/ou liberado na atmosfera pelos ecossistemas florestais (Sanquetta, 2002).

As metodologias utilizadas para quantificação de biomassa florestal dividem-se em métodos diretos (destrutivos) e métodos indiretos (não destrutivos). Os diretos significam uma medição real direta da biomassa, como, por exemplo, a pesagem de um fuste inteiro por meio de um dinamômetro ou uma balança. Todas as árvores de uma determinada parcela são derrubadas e pesadas, sendo efetuada, em seguida, a extrapolação da avaliação amostrada para a área total de interesse.

A predição de biomassa aérea pelo método indireto consiste em correlacioná-la com alguma variável de fácil obtenção e que não requeira a destruição do material vegetal. As estimativas podem ser obtidas por meio de relações quantitativas ou matemáticas, como razões ou regressões de dados provenientes de inventários florestais (diâmetro à altura do peito - DAP, altura e volume) ou por dados de sensoriamento remoto (imagens de satélite) (Higuchi \& Carvalho Junior, 1994). Estes dois métodos ainda são bastante discutidos entre os autores, que dividem suas opiniões sobre qual a melhor forma de se estimar a biomassa. Neste sentido, sabe-se que independente do método a ser utilizado (direto ou indireto), seja árvore individual ou por unidade de área, as estimativas podem fornecer incertezas devido a todos os fatores que exercem influência sobre as predições (Sanquetta, 2002; Sanquetta e Balbinot, 2004; Vismara, 2010).
Oúnico consenso é que é inviável determinar a biomassa de cada árvore pelo método direto. Por isso é necessário realizar as análises de regressão para ajustar modelos estatísticos que possam ser aplicados para estimar biomassa em regiões maiores (Fearnside et al., 1993; Higuchi et al., 1998).

Segundo Nelson et al. (1999), é possível melhorar as estimativas de carbono sequestrado por florestas utilizando equações empíricas preexistentes na literatura. Porém, estas equações precisam ser aplicadas corretamente, considerando, por exemplo, o mesmo tipo de floresta de onde foram originadas e parâmetros biométricos dentro do intervalo daqueles usados para desenvolver a equação (amplitude de DAP e altura). O estudo mostrou que, quando critérios como estes não são respeitados, as estimativas de biomassa por equações empíricas podem gerar erros de até $60 \%$ em relação ao valor esperado.

Desta forma, visando aprimorar as estimativas de biomassa florestal por métodos não destrutivos e, percebendo que existem no mercado inúmeros dendrômetros ópticos capazes de cubar as árvores em pé, objetivou-se neste trabalho verificar a acurácia de dois dendrômetros ópticos na cubagem de árvores por métodos não destrutivos e estimar a influência que a medição de variáveis com esses instrumentos possui sobre a predição da biomassa florestal por meio de modelos estocásticos disponíveis na literatura florestal.

\section{Material e Métodos}

\section{Área de estudo}

$\mathrm{O}$ estudo foi realizado na Estação Experimental de Ciências Florestais de Itatinga do Departamento de Ciências Florestais da Universidade de São Paulo (ESALQ/USP), localizada na região centro sul do Estado de São Paulo, a $23^{\circ} 10^{\prime} \mathrm{S}$ e $48^{\circ} 40^{\prime} \mathrm{W}$, com 857 metros de altitude média. $\mathrm{O}$ clima da região, segundo Köeppen, é caracterizado como mesotérmico úmido (Cwa), com precipitação anual média de $1.308 \mathrm{~mm}$. A temperatura média do mês mais frio é $12,8^{\circ} \mathrm{C}$ e a média do mês mais quente, $19,4{ }^{\circ} \mathrm{C}$.

$\mathrm{O}$ experimento foi realizado em um povoamento de Eucalyptus grandis Maiden com 13 anos de idade e espaçamento $3 \mathrm{~m} \times 2 \mathrm{~m}$. As medições foram feitas em três unidades amostrais de $360 \mathrm{~m}^{2}$ cada, com cerca de 60 árvores por parcela. Desta forma, foram mensuradas 175 árvores nesse povoamento. Um resumo dos dados relativos às três parcelas pode ser encontrado na Tabela 1. 
Tabela 1. Resumo dos dados de DAP, altura total, volume total, umidade e biomassa total média por árvore para as três parcelas do povoamento de Eucalyptus grandis.

\begin{tabular}{cccccccccc}
\hline \multirow{2}{*}{ Parcela } & \multicolumn{3}{c}{ DAP $(\mathbf{c m})$} & \multicolumn{3}{c}{$\mathbf{h}(\mathbf{m})$} & \multicolumn{3}{c}{$\mathbf{v}\left(\mathbf{m}^{3}\right)$} \\
\cline { 2 - 9 } & mín. & médio & máx. & mín. & médio & máx. & mín. & médio & máx. \\
\hline 1 & 10,0 & 15,9 & 20,8 & 15,7 & 25,3 & 28,4 & 0,053 & 0,246 & 0,458 \\
2 & 10,4 & 15,3 & 20,4 & 19,4 & 24,9 & 28,6 & 0,079 & 0,226 & 0,439 \\
3 & 10,9 & 15,2 & 19,0 & 19,0 & 24,8 & 28,5 & 0,093 & 0,219 & 0,367 \\
\hline \multirow{2}{*}{ Parcela } & \multicolumn{3}{c}{ Umidade (\%) } & \multicolumn{3}{c}{ Biomassa (kg) } & & & \\
\cline { 2 - 7 } & mín. & médio & máx. & mín. & médio & máx. & & & \\
2 & 34,9 & 45,8 & 51,1 & 30,4 & 151,8 & 285,3 & & & \\
3 & 41,1 & 47,5 & 59,1 & 43,7 & 123,5 & 247,8 & & & \\
\hline
\end{tabular}

Sendo que: “mín." e "máx." representam, respectivamente, o valor mínimo e máximo da variável de interesse observada na parcela correspondente. A variável biomassa foi determinada pela técnica gravimétrica.

\section{Cubagem rigorosa}

As medidas dos diâmetros a diferentes alturas foram tomadas a $0,1 \mathrm{~m} ; 0,4 \mathrm{~m} ; 0,7 \mathrm{~m} ; 1,0 \mathrm{~m} ; 1,3 \mathrm{~m} ; 2,0 \mathrm{~m}$ e em intervalos de $1,0 \mathrm{~m}$ até a altura de $8,0 \mathrm{~m}$, totalizando doze diâmetros por indivíduo. Este procedimento foi efetuado com os dendrômetros Criterion 400 e RC3H, sob forma não destrutiva. Para medir os diferentes diâmetros com o RC3H usava-se a vara telescópica para delimitar a altura desejada. Utilizou-se a vara telescópica apenas com o $\mathrm{RC} 3 \mathrm{H}$ porque o mesmo não consegue determinar a altura em que vai realizar a medição do diâmetro no tronco. Os diâmetros foram medidos até uma altura de $8,0 \mathrm{~m}$ pois, em levantamentos preliminares, percebeu-se que acima deste ponto, os diâmetros obtidos apresentavam grande diferença com o verdadeiro.

O Criterion modelo 400 considerado por Liu et al. (1993) como a versão digital do Relascópio de Bitterlich, pode ser usado para medir: diâmetro a qualquer altura, alturas das árvores; área basal do povoamento; inclinação do terreno em graus e em porcentagem; distâncias horizontais e inclinadas; ângulos horizontais; coordenadas para levantamentos topográficos. O RC3H é um dendrômetro digital da Savcor Masser que possui um holograma especial para maior exatidão na contagem eletrônica de ângulos exatos. Corrige automaticamente a inclinação do terreno, impermeável e possibilita a medição de altura das árvores, inclinação, distância horizontal e diâmetros em diferentes alturas.

Depois de mensurados os diâmetros dos indivíduos em pé com os dois dendrômetros, cada árvore foi numerada com placa metálica e derrubada com motossera. A cubagem rigorosa foi realizada com auxílio de trena e suta. Foram marcadas ao longo do fuste as mesmas alturas de medição quando da cubagem em pé, além da altura total. Após os $8,0 \mathrm{~m}$ seguiram-se intervalos de $1,0 \mathrm{~m}$ nas seções do tronco até que o diâmetro fosse menor que $5,0 \mathrm{~cm}$. Para os diâmetros obtidos com a suta, foram efetuadas duas medições perpendiculares entre si em cada diâmetro e obtida sua média.

\section{Volume das árvores}

$\mathrm{O}$ volume individual das árvores foi calculado pela fórmula de Smalian conforme Machado \& Figueiredo Filho (2006). Desta forma, obteve-se o volume com variáveis oriundas de três métodos: do uso do Criterion 400 , do $\mathrm{RC} 3 \mathrm{H}$ e do volume considerado verdadeiro através da cubagem rigorosa. Para obter o volume a partir dos $8,0 \mathrm{~m}$ pelo Criterion 400 e pelo $\mathrm{RC} 3 \mathrm{H}$ usou-se o fator de forma calculado da seguinte forma:

$$
f_{i}=\frac{v \cdot \text { ponta }_{i}}{\left(\frac{\pi \cdot d_{g i}^{2}}{40000}\right) \cdot\left(h t_{i}-8\right)}
$$

Onde: $i=$ é o índice que se refere às árvores; $v \cdot p_{0}$ ton $a_{i}=$ é a diferença entre o volume rigoroso total da árvore $\left(\mathrm{m}^{3}\right)$ e o volume rigoroso até $8,0 \mathrm{~m}$ da árvore $\left(\mathrm{m}^{3}\right) ; d_{8 i}=$ diâmetro a $8,0 \mathrm{~m}$ de altura da cubagem rigorosa da árvore $(\mathrm{cm})$; $\mathrm{h}_{\mathrm{ti}}=$ altura total da árvore $(\mathrm{m})$.

Após o cálculo do fator de forma, determinou-se os volumes a partir de $8,0 \mathrm{~m}$ até a altura total para os métodos do Criterion 400 e RC3H usando-se a seguinte equação: 


$$
v p_{i}=\frac{\pi \cdot d_{g i}^{2}}{40000} \cdot\left(h t_{i}-8\right) \cdot f_{i}
$$

Onde: $i=$ é o índice que se refere às árvores; $d_{8 i}=$ diâmetro a $8,0 \mathrm{~m}$ de altura da cubagem rigorosa da árvore $(\mathrm{cm}) ; h_{t i}=$ altura total da árvore $(\mathrm{m}) ; f_{i}=$ fator de forma da árvore.

Logo, os volumes totais pelo Criterion $400 \mathrm{e} \mathrm{RC} 3 \mathrm{H}$ foram obtidos pela soma dos volumes das seções até $8,0 \mathrm{~m}$ de altura e pelo volume da ponta $(v p)$.

\section{Discos de madeira amostrados do tronco}

Foram retirados de cada indivíduo discos em quatro posições relativas referentes à altura total da árvore: a $0 \%$ (disco da base), altura do DAP, $50 \%$ e $100 \%$ da altura total, sendo considerado até o limite mínimo de $5 \mathrm{~cm}$. Os discos foram pesados após o corte no campo, para determinação do peso úmido $(M u)$, em balança analítica com precisão de um grama.

\section{Determinação do teor de umidade}

Para determinação do teor de umidade, os discos foram secos em estufa de circulação forçada de ar a 103 ${ }^{\circ} \mathrm{C} \pm 2{ }^{\circ} \mathrm{C}$ até peso constante $(M s)$. Após a secagem, o teor de umidade das amostras $(U)$ foi determinado pela seguinte fórmula:

$$
U_{i}=\frac{\left(M_{u}-M_{s}\right)}{M_{u}} .100
$$

Em que: $i$ é o índice que se refere às árvores; $U$ é o teor de umidade das amostras (\%); $M u$ é a massa úmida das amostras (kg); Ms é a massa seca das amostras ( $\mathrm{kg})$.

\section{Determinação da biomassa}

Para obtenção da biomassa aérea das árvores individuais foram utilizadas as técnicas gravimétrica e volumétrica.

a) Técnica gravimétrica

A massa verde do tronco foi medida com auxílio de uma balança analítica, com as toras seccionadas no campo. A biomassa foi obtida da seguinte forma:

$$
b_{g i}=m_{i} \cdot\left(1-\frac{U_{i}}{1000}\right)
$$

Onde: $i$ é o índice que se refere às árvores; $b_{g}$ é a biomassa do tronco pela técnica gravimétrica $(\mathrm{kg}) ; m$ é a massa verde do tronco $(\mathrm{kg})$; $U$ é o teor de umidade do tronco $(\%)$. b) Técnica volumétrica

Após obter-se o volume sólido das árvores por meio da cubagem rigorosa pelo método de Smalian, a biomassa do tronco foi determinada através da seguinte fórmula:

$$
b_{v i}=v_{i} \cdot d
$$

Onde: $i$ é o índice que se refere às árvores; $b_{v}$ é a biomassa do tronco pela técnica volumétrica $(\mathrm{kg}) ; v$ é o volume do tronco obtido pela cubagem $\left(\mathrm{m}^{3}\right)$ para cada um dos três métodos (cubagem tradicional, Criterion e $\mathrm{RC} 3 \mathrm{H}) ; d$ é a densidade básica média da espécie entre todos os indivíduos amostrados, equivalente a $0,5724 \mathrm{~g} \mathrm{~cm}^{-3}$.

Sabe-se que a densidade pode variar entre espécies, indivíduos e dentro da mesma árvore, tanto no sentido longitudinal (base-topo) como no sentido radial da medula para a casca (Panshin \& De Zeew, 1980). Porém, neste trabalho utilizou-se um valor médio entre todos os indivíduos.

\section{Modelos de predição de biomassa do tronco sob a técnica gravimétrica}

Alguns modelos foram selecionados da literatura para testar o ajuste da biomassa do fuste obtida pela técnica gravimétrica (Tabela 2).

No caso dos modelos com ajustes logarítmicos, ocorre uma discrepância devido à transformação matemática, ao se efetuar a operação inversa para se obter a variável de interesse. A correção para essa discrepância foi feita multiplicando-se a variável dependente encontrada pelo Fator de Correção de Meyer (Machado et al., 2008).

\section{Modelos de predição de biomassa do tronco sob a técnica volumétrica}

Para a técnica volumétrica foram ajustados três modelos (Tabela 3), sendo que esses foram os que tiveram o melhor ajuste pela técnica gravimétrica.

Todos os modelos para as diferentes técnicas, com seus respectivos coeficientes de regressão podem ser visualizados em Nicoletti (2011).

\section{Ajuste e Avaliação dos modelos de predição}

Para selecionar o melhor modelo empírico para as diferentes técnicas de determinação da biomassa utilizaram-se os seguintes critérios:

a) Critério de informação Akaike (AIC)

O AIC é uma metodologia simples e efetiva para a seleção de modelos para análise de dados empíricos 
por meio da relação da distância entre os dois modelos, chamada de Kulbach-Leibler, e a função de máxima logverossimilhança de Fisher (Burnham e Anderson, 2002). Este critério estima a discrepância geral relativa entre um modelo de aproximação e o modelo real (hipotético) que gerou os dados. Desta forma, quanto menor o valor do
AIC de um modelo, menor sua discrepância geral estimada e, por consequência, menor sua distância relativa para o modelo real (hipotético). A fórmula para o cálculo do AIC é: $A I C=-2 \ln (m v)+2 p$

Em que: $m v$ é o valor de maior máxima verossimilhança apresentada pelo modelo; $p$ é o número de parâmetros.

Tabela 2. Modelos empíricos para determinação da biomassa através da técnica gravimétrica.

\begin{tabular}{ccc}
\hline Equação & Modelo Empírico & Autores \\
\hline 1 & $\ln \mathrm{b}_{g i}=\mathrm{b}_{0}+\mathrm{b}_{1} \ln \left(\mathrm{d}^{2} \mathrm{~h}\right)$ & Spurr \\
2 & $\mathrm{~b}_{g i}=\mathrm{b}_{0}+\mathrm{b}_{1}\left(\mathrm{~d}^{2} \mathrm{~h}\right)$ & Brown \\
3 & $\mathrm{~b}_{g i}=\mathrm{b}_{0}+\mathrm{b}_{1} \mathrm{~d}^{2}+\mathrm{b}_{2} \mathrm{~d}^{2} \mathrm{~h}+\mathrm{b}_{3} \mathrm{~h}$ & Stoate \\
4 & $\mathrm{~b}_{g i}=\mathrm{b}_{0}+\mathrm{b}_{1} \mathrm{~d}+\mathrm{b}_{2} \mathrm{~d}^{2}+\mathrm{b}_{3} \mathrm{dh}+\mathrm{b}_{4} \mathrm{~d}^{2} \mathrm{~h}+\mathrm{b}_{5} \mathrm{~h}$ & Meyer \\
5 & $\mathrm{~b}_{g i}=\mathrm{b}_{0}+\mathrm{b}_{1} \mathrm{~d}^{2}+\mathrm{b}_{2} \mathrm{~d}^{2} \mathrm{~h}+\mathrm{b}_{3} \mathrm{dh}^{2}+\mathrm{b}_{4} \mathrm{~h}^{2}$ & Naslund modificada \\
6 & $\ln \mathrm{b}_{g i}=\mathrm{b}_{0}+\mathrm{b}_{1} \ln (\mathrm{d})+\mathrm{b}_{2} \ln (\mathrm{h})$ & Schumacher-Hall \\
7 & $\ln \mathrm{b}_{g i}=\mathrm{b}_{0}+\mathrm{b}_{1} \ln (1 / \mathrm{d})$ & Schumacher \\
\hline
\end{tabular}

Sendo que: $b_{g i}$ é a biomassa obtida pela técnica gravimétrica $(\mathrm{kg}) ; d$ é o diâmetro a $1,30 \mathrm{~m}$ das árvores $(\mathrm{cm})$; $h$ é a altura total (m) e $b_{0}, b_{1,}, b_{2}, b_{3}, b_{4}$ e $b_{5}$ são os coeficientes da regressão. Fonte: Schneider (1998).

Tabela 3. Modelos empíricos para determinação da biomassa por meio da técnica volumétrica.

\begin{tabular}{ccc}
\hline Equação & Modelo Empírico & Autores \\
\hline 1 & $\ln \mathrm{b}_{v i}=\mathrm{b}_{0}+\mathrm{b}_{1} \ln \left(\mathrm{d}^{2} \mathrm{~h}\right)$ & Spurr \\
2 & $\ln \mathrm{b}_{v i}=\mathrm{b}_{0}+\mathrm{b}_{1} \ln (\mathrm{d})+\mathrm{b}_{2} \ln (\mathrm{h})$ & Schumacher-Hall \\
3 & $\ln \mathrm{b}_{v i}=\mathrm{b}_{0}+\mathrm{b}_{1} \ln (1 / \mathrm{d})$ & Schumacher \\
\hline
\end{tabular}

Sendo que: $b_{v v}$ é a biomassa obtida pela técnica volumétrica $(\mathrm{kg}) ; d$ é o diâmetro a $1,30 \mathrm{~m}$ das árvores $(\mathrm{cm}) ; h$ é a altura total (m) e $b_{0}, b_{l}, b_{2}$ são os coeficientes da regressão. Fonte: Schneider (1998).

b) Coeficiente de determinação ajustado $\left(\mathrm{R}^{2}{ }_{\mathrm{aj}}\right)$

$\mathrm{O}$ coeficiente de determinação $\left(\mathrm{R}^{2}\right)$ expressa a variabilidade do modelo explicada pela regressão. Como o coeficiente de determinação cresce à medida que se inclui uma nova variável ao modelo matemático, foi utilizado o coeficiente de determinação ajustado $\left(\mathrm{R}^{2}\right.$ aj. $)$ que pondera o número de coeficientes da equação, obtido pela fórmula a seguir:

$$
R_{a j .}^{2}=R^{2}-\left(\frac{p-1}{n-p}\right) \cdot\left(1-R^{2}\right)
$$

Sendo: $R^{2}=$ coeficiente de determinação; $p=$ número de coeficientes da equação (parâmetros); $n=$ número de observações.

Quanto mais próximos de 1 forem os valores dos coeficientes de determinação, melhor será o ajuste da linha de regressão. Assim, por este critério, o melhor modelo matemático seria o que apresentasse o maior valor de $\mathrm{R}^{2}{ }_{\mathrm{aj}}$.

c) Erro padrão da estimativa $\left(\mathrm{S}_{\mathrm{yx}}\right)$

$\mathrm{O}$ erro padrão da estimativa também foi utilizado como critério na seleção dos modelos, segundo a equação abaixo:

$$
S_{y x}=\sqrt{\frac{\sum_{i=1}^{n}(Y-\hat{Y})^{2}}{n-p}}
$$

Onde: $S_{y x}=$ erro padrão da estimativa na unidade da variável resposta; $Y$ é a variável regressora estimada pelo modelo; $Y$ é a variável observada; $n=$ número de observações; $p$ é o número de parâmetros. 
Assim, quanto menor for o erro padrão da estimativa, melhores são as estimativas obtidas na equação.

d) Erro padrão da estimativa relativo $\left(S_{y x \%}\right)$

O $S_{y x \%}$ é obtido pela fórmula:

$$
S_{y x \%}=\frac{S_{y x}}{\bar{y}} .100
$$

Sendo: $S_{y x}=$ erro padrão da estimativa absoluto; $S_{v x \%}=$ erro padrão da estimativa em porcentagem; $y$ é a média aritmética da variável dependente.

Sabe-se que quanto menor o valor do $S_{y x \%}$ melhor é o ajuste fornecido pelo modelo. Assim, analisou-se este parâmetro juntamente com todos os demais para a seleção da equação.

e) Análise gráfica do erro de predição relativo

Analisaram-se os gráficos dos resíduos existentes na predição da biomassa do fuste por meio da técnica volumétrica e através do ajuste dos melhores modelos. Os diferentes métodos de cubagem não destrutivos, os provenientes do $\mathrm{RC} 3 \mathrm{H}$ e do Criterion, puderam ser confrontados com a biomassa obtida com a técnica gravimétrica que foi a considerada verdadeira. Com a diferença entre a biomassa da técnica gravimétrica e com os métodos não destrutivos, seja pela predição dos modelos ou pela técnica volumétrica, foi possível avaliar a magnitude do erro na predição da biomassa das árvores.

\section{Resultados e Discussão}

\section{Análise descritiva das variáveis utilizadas para a predição}

As estatísticas descritivas da variável dependente (biomassa obtida por gravimetria e biomassa obtida por volumetria) utilizadas no ajuste dos modelos são descritas na Tabela 4. Nesta tabela são descritas a técnica utilizada (volumétrica, predição pelos modelos e gravimétrica) com os respectivos métodos (cubagem tradicional, Criterion e $\mathrm{RC} 3 \mathrm{H})$. Percebe-se que, independentemente da técnica utilizada, seja volumétrica ou com a predição da mesma, a biomassa obteve estimativas semelhantes para o mesmo método. Comparando-se a técnica gravimétrica, considerada a mais exata na determinação da biomassa, com as outras estimativas, nota-se que a cubagem tradicional, independentemente da técnica, fornece valores muito próximos aos considerados reais, sendo que o Criterion e o $\mathrm{RC} 3 \mathrm{H}$ forneceram estimativas subestimadas desta variável. As estimativas obtidas com o Criterion foram mais exatas do que as com o $\mathrm{RC} 3 \mathrm{H}$.

Através do comportamento do desvio padrão da estimativa pode-se dizer que as menores variações foram apresentadas usando o método do dendrômetro da $\mathrm{RC} 3 \mathrm{H}$. Estas indicam que esse método fornece estimativas precisas, porém, não exatas. O coeficiente de variação teve comportamento praticamente constante entre as diferentes técnicas testadas, variando de $32,7 \%$ até $37,4 \%$.

Tabela 4. Estatísticas descritivas da variável dependente utilizada na construção dos modelos para predizer a biomassa do tronco.

\begin{tabular}{|c|c|c|c|c|c|c|}
\hline \multirow{2}{*}{ Técnica } & \multirow{2}{*}{ Método } & \multicolumn{5}{|c|}{ Biomassa (kg) } \\
\hline & & Mín. & Média & Máx. & $\mathrm{s}$ & $\mathrm{CV}(\%)$ \\
\hline \multirow{3}{*}{ Volumétrica } & Cubagem tradicional & 30,4 & 131,6 & 262,2 & 46,3 & 35,17 \\
\hline & Criterion & 24,8 & 115,8 & 268,0 & 43,4 & 37,45 \\
\hline & $\mathrm{RC} 3 \mathrm{H}$ & 18,4 & 78,1 & 186,6 & 28,1 & 35,93 \\
\hline \multirow{3}{*}{$\begin{array}{c}\text { Predição } \\
\text { com os modelos } \\
\text { selecionados }\end{array}$} & Cubagem tradicional & 32,8 & 131,4 & 252,1 & 45,3 & 34,47 \\
\hline & Criterion & 29,9 & 114,3 & 215,2 & 38,2 & 33,44 \\
\hline & $\mathrm{RC} 3 \mathrm{H}$ & 22,4 & 77,5 & 145,3 & 25,3 & 32,69 \\
\hline Gravimétrica & - & 30,4 & 131,5 & 285,3 & 46,9 & 35,64 \\
\hline
\end{tabular}

Sendo que: "mín." é o valor mínimo, "máx." é o valor máximo, "s" é o desvio padrão e " $C V$ " é o coeficiente de variação das observações. 


\section{Critérios de seleção dos modelos ajustados por meio da técnica gravimétrica}

Os critérios de seleção dos modelos estudados através da técnica gravimétrica podem ser visualizados na Tabela 5.

Tabela 5. Valores dos critérios de seleção dos modelos analisados para a Técnica Gravimétrica.

\begin{tabular}{ccccc}
\hline Modelo & $\mathbf{R}_{\text {aj. }}{ }^{2}$ & $\mathrm{~S}_{\mathrm{yx} \%}$ & Erro & AIC \\
\hline Spurr & $\mathbf{0 , 9 2 5 7}$ & $\mathbf{1 6 , 1 5}$ & $\mathbf{1 3 , 3 7}$ & $\mathbf{1 3 4 4 , 5}$ \\
Brown & 0,9033 & 11,09 & 14,57 & 1430,2 \\
Stoate & 0,9031 & 11,10 & 14,59 & 1432,4 \\
Meyer & 0,9039 & 11,05 & 14,53 & 1432,9 \\
$\begin{array}{c}\text { Naslund } \\
\text { modificada }\end{array}$ & 0,9030 & 11,10 & 14,59 & 1433,6 \\
Schumacher-Hall & 0,9255 & 16,35 & 13,39 & 1348,8 \\
Schumacher & 0,8980 & 19,41 & 15,65 & 1081,2 \\
\hline
\end{tabular}

Sendo que, $R_{a j}^{2}$ é o coeficiente de determinação ajustado, $S_{y x}$ é o erro padrão relativo da predição (\%), Erro é o erro padrão absoluto da predição encontrado pelo modelo e $A I C$ é o Critério de informação de Akaike.

Os resultados mostraram que, de modo geral, os modelos apresentaram altos coeficientes de determinação empírico, sendo eles superiores a 0,8980 . Os melhores valores encontrados para o $\mathrm{R}_{\text {aj. }}^{2}$ foram referentes à equação de Spurr $(0,9257)$ e a equação de Schumacker-Hall $(0,9255)$. Os demais apresentaram valores bem próximos de $\mathrm{R}_{\mathrm{aj}}^{2}$, entre 0,8980 e 0,9039 . O erro padrão absoluto da predição não teve grande variação $(13,37$ até 15,65$)$ entre os modelos estudados, sendo o de Spurr o que apresentou o menor valor. Silva et al. (2004), testando diferentes modelos para predizer a biomassa do tronco em povoamentos de Eucalyptus sp., também encontraram o menor erro padrão para o modelo que utilizou a variável combinada $\left(\mathrm{d}^{2} \mathrm{~h}\right)$. Vismara et al. (2010) verificaram grandes problemas na predição utilizando seis equações de biomassa e de carbono em áreas distintas onde estas foram ajustadas. A avaliação segundo estes autores foi que a maioria das equações avaliadas teve desempenho muito aquém da equação referência, demonstrando a pouca generalidade de sua aplicação em áreas distintas aonde elas foram obtidas.

Para os valores de AIC, o modelo de Schumacher foi aquele que demonstrou o melhor ajuste (AIC $=1081,2)$. O modelo de Spurr $(\mathrm{AIC}=1344,5)$ e o de Schumacher-Hall $(\mathrm{AIC}=1348,8)$ também obtiveram bom ajuste em relação aos demais, sendo identificados como os melhores modelos testados. Através do AIC nota-se que aqueles que apresentaram a escala logarítmica no ajuste, forneceram as melhores estimativas. Apenas com relação ao erro padrão relativo estes modelos apresentaram estimativas piores que os demais. Entretanto, a combinação de todos os índices ainda destaca os três modelos como sendo os que melhor explicam a variável predita. O modelo selecionado para as predições foi o de Spurr (Tabela 5).

\section{Critérios de seleção dos modelos ajustados através da técnica volumétrica}

Através dos critérios de seleção dos modelos abordados (Tabela 6), selecionou-se o melhor modelo por meio do ajuste com a técnica volumétrica.

Tabela 6. Valores dos critérios de seleção dos modelos analisados para a Técnica Volumétrica.

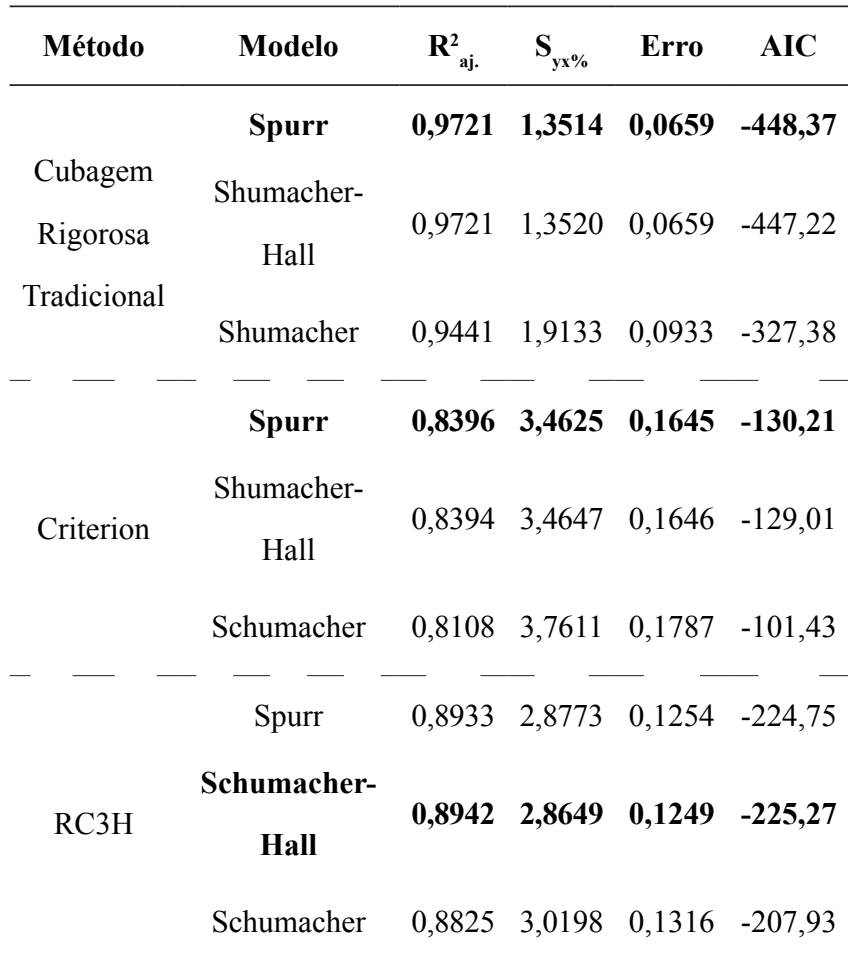

Sendo que, $R_{a j}^{2}$ é o coeficiente de determinação ajustado, $S_{y x^{\circ} \%}$ é o erro padrão relativo (\%), Erro é o erro padrão absoluto de predição encontrado pelo modelo e $A I C$ é o Critério de informação de Akaike.

Os critérios de seleção dos três modelos ajustados com a técnica volumétrica permitem afirmar que o coeficiente de determinação ajustado obteve a mesma tendência de ajuste para os diferentes métodos testados. Para todos os casos o ajuste demonstrou altos valores para o coeficiente de determinação, variando de 0,8108 até 0,9721 , sendo que 
os modelos com variáveis oriundas de medições usando o Criterion foram os que apresentaram os menores valores, seguidos daqueles relativos ao $\mathrm{RC} 3 \mathrm{H}$ e, após, os com a cubagem rigorosa. Pode-se observar que tanto para o método da cubagem rigorosa, do Criterion e do $\mathrm{RC} 3 \mathrm{H}$ o modelo de Spurr e o de Schumacher-Hall tiveram as maiores e melhores estimativas para todos os critérios analisados. Nestes modelos praticamente não houve diferença entre os índices avaliados. Para o AIC houve essa mesma tendência, dos modelos de Spurr e do Schumacher-Hall, em apresentar os menores e melhores valores, com pouca diferença entre eles. Aúnica exceção existente foi para o método com o $\mathrm{RC} 3 \mathrm{H}$, em que o modelo de Shumacher-Hall apresentou valor de AIC e $\mathrm{R}^{2}{ }_{\text {aj }}$ um pouco maior. Diante destes, percebe-se que a introdução nos modelos da altura, como segunda variável independente, melhorou o ajuste. Comportamento semelhante a este foi encontrado por Silveira (2009) ao ajustar diversos modelos aritméticos e logarítmicos. Este fato sugere que a adição de uma segunda variável independente no modelo melhora sua capacidade de explicação biológica da biomassa.

O erro padrão relativo e absoluto da predição apresentaram a mesma tendência nos três métodos. Os dados fornecidos pela cubagem apresentaram os melhores valores de erro padrão, seguidos daqueles pelo $\mathrm{RC} 3 \mathrm{H}$ e após os com o Criterion.

Erros de predição da biomassa com os diversos métodos de ajuste

$\mathrm{Na}$ tabela 7 são apresentados os modelos que apresentaram as melhores estimativas para realizar a predição e verificar seus respectivos erros.

Leite e Andrade (2004) estudaram o método da altura relativa que como o contexto deste trabalho, desejouse encontrar metodologias alternativas ao tradicional, propondo modelar o taper e o volume de árvores tanto de povoamentos equiâneos ou de inequiâneos. Assim, por meio das avaliações efetuadas, concluíram que o método altura relativa resultou em estimativas precisas e não tendenciosas do taper e do volume sólido, podendo ser utilizado em substituição ao usual.

O erro relativo de predição da biomassa (\%) em função do DAP $(\mathrm{cm})$ em relação à biomassa obtida pela técnica gravimétrica pode ser visualizado na Figura 1. A média do erro de predição apresentado por meio dos dados provenientes da cubagem rigorosa $(-0,66 \%)$ e com aqueles resultantes da predição dos modelos $(-0,94 \%)$ apresentou valores muito próximos de zero. Este fato já era esperado, pois a cubagem, se bem realizada, conduz a estimativas com grande confiabilidade. Obteve-se um desvio padrão desta predição igual a 8,3\% para a cubagem convencional e 11,3\% para o modelo. Este resultado confirma que a distribuição mais uniforme foi apresentada na Figura 1 (A), onde as observações de modo geral variam de $\pm 10 \%$ com variância homogênea. Porém, em ambos os casos, a média das observações teve comportamento semelhante com o aumento do DAP das árvores. De modo geral, a média foi levemente superestimada, com exceção do intervalo entre aproximadamente $14 \mathrm{~cm}$ e $16 \mathrm{~cm}$ do diâmetro, quando demonstrou estar sendo subestimada.

Analisando a distribuição dos erros de predição para o Criterion (Figura 2) percebe-se o mesmo comportamento quando feita a cubagem pelo $\mathrm{RC} 3 \mathrm{H}$ da Masser (Figura 3), ou seja, em que a melhor distribuição dos resíduos foi encontrada quando realizado o ajuste pelo melhor modelo. Este fato pode ser comprovado pelos valores do desvio padrão desse erro, sendo $13,4 \%$ na forma não destrutiva e 9,9\% com o ajuste. As médias, para este caso, foram superestimadas em torno de $11 \%$. Este dendrômetro também tendeu a fornecer medidas mais

Tabela 7. Modelos selecionados para predição da biomassa do tronco das árvores sob a Técnica Gravimétrica e a Volumétrica.

\begin{tabular}{ccc}
\hline Técnica & Método & Modelo Matemático \\
\hline \multirow{2}{*}{ Volumétrica } & Cubagem Rigorosa & $\ln \mathrm{b}=-3,9193+1,0069 \ln \left(\mathrm{d}^{2} \mathrm{~h}\right)$ \\
\hline Criterion & $\ln \mathrm{b}=-3,7655+0,9736 \ln \left(\mathrm{d}^{2} \mathrm{~h}\right)$ \\
\hline Gravimétrica & $\mathrm{RC} 3 \mathrm{H}$ & $\ln \mathrm{b}=-3,4674+2,0234 . \ln (\mathrm{d})+0,6964 \ln (\mathrm{h})$ \\
\hline
\end{tabular}

Sendo que, $b$ é a biomassa do fuste $(\mathrm{kg}), d$ é o diâmetro à altura do peito $(\mathrm{cm}), h$ é a altura total $(\mathrm{m})$ e $l n$ é o logaritmo natural. 
precisas para os indivíduos de menor porte. Williams et al. (1999) avaliando equações de taper ajustadas com dados resultantes de dois dendrômetros ópticos, Barr \& Stroud e do Criterion 400, notaram que houve diferença significativa apenas para aquelas geradas com o Criterion.

$\mathrm{O}$ dendrômetro $\mathrm{RC} 3 \mathrm{H}$ da Masser forneceu um erro de predição médio subestimado de praticamente $40 \%$ tanto na cubagem não destrutiva, como pelo modelo selecionado. $\mathrm{O}$ erro foi diretamente proporcional ao diâmetro até cerca dos $16 \mathrm{~cm}$, quando tendeu a ser constante com o aumento do tamanho dos indivíduos. Ou seja, para as árvores de menor DAP o equipamento tende a fornecer medidas mais precisas. Porém, de modo geral não houve uma tendência na predição da biomassa do fuste pelos métodos testados, o que caracteriza novamente variância homogênea. Através da distribuição das observações, percebe-se um comportamento contrário àquele observado com os dados da cubagem rigorosa tradicional. A distribuição dos resíduos na Figura 3 (B) teve comportamento mais uniforme que aquela obtida pela cubagem não destrutiva, fato este comprovado pelos valores do desvio padrão de $9,3 \%$ para a forma não destrutiva e de $7,0 \%$ com o ajuste do modelo. Soares et al. (2005) obtiveram a mesma tendência do erro padrão residual percentual, ou seja, sem uma tendência na estimação dos estoques de carbono do tronco em povoamentos de eucaliptos em equações com as variáveis independentes idade e índice de sítio.
A

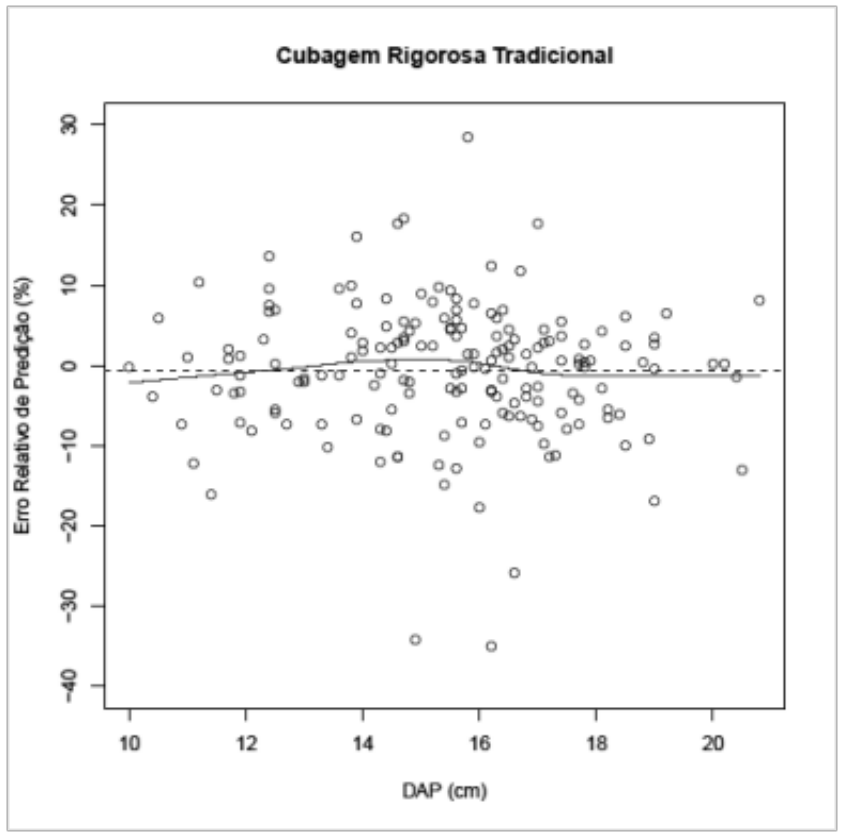

B

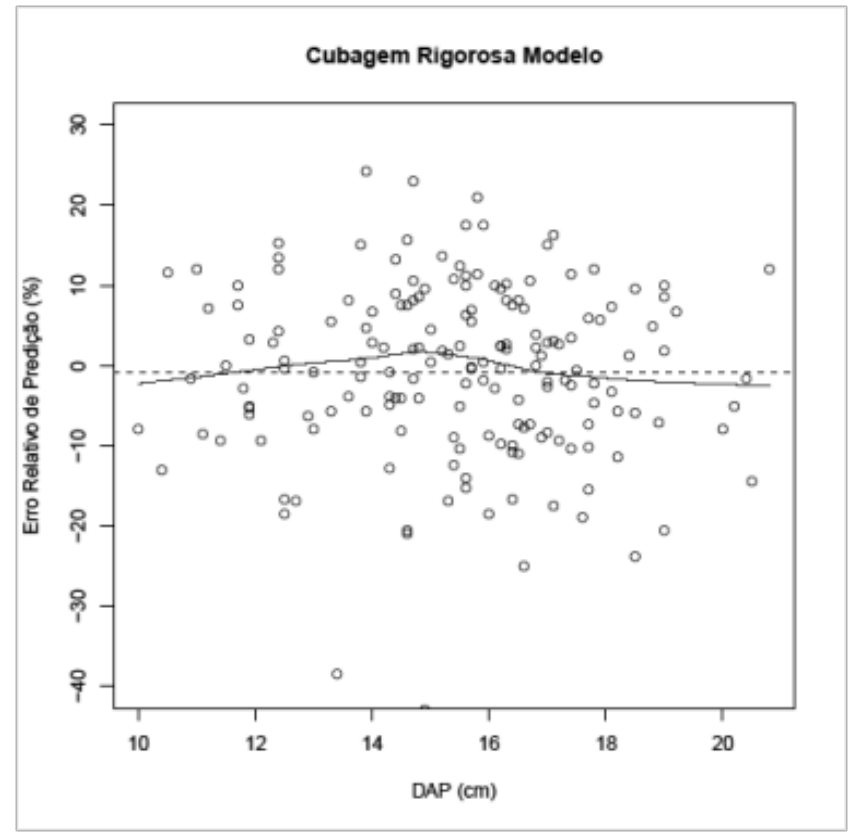

Figura 1 . (A) Erro relativo de predição (\%) da cubagem rigorosa tradicional em função do DAP (cm) e (B) com os dados da Cubagem Rigorosa por meio do melhor modelo ajustado em função do DAP $(\mathrm{cm})$. 
A

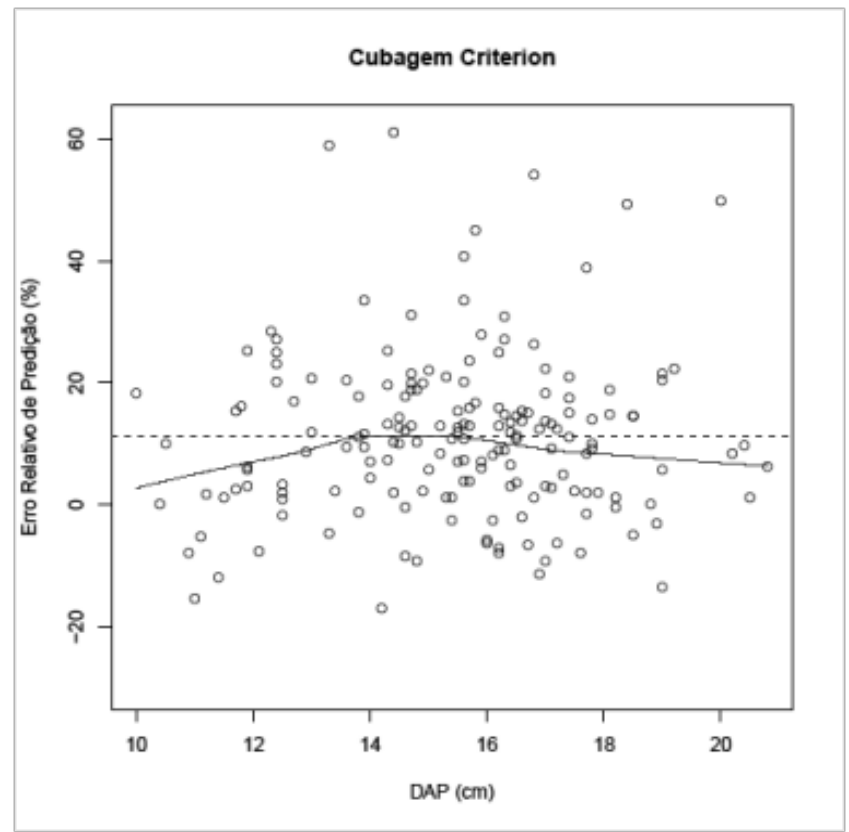

B

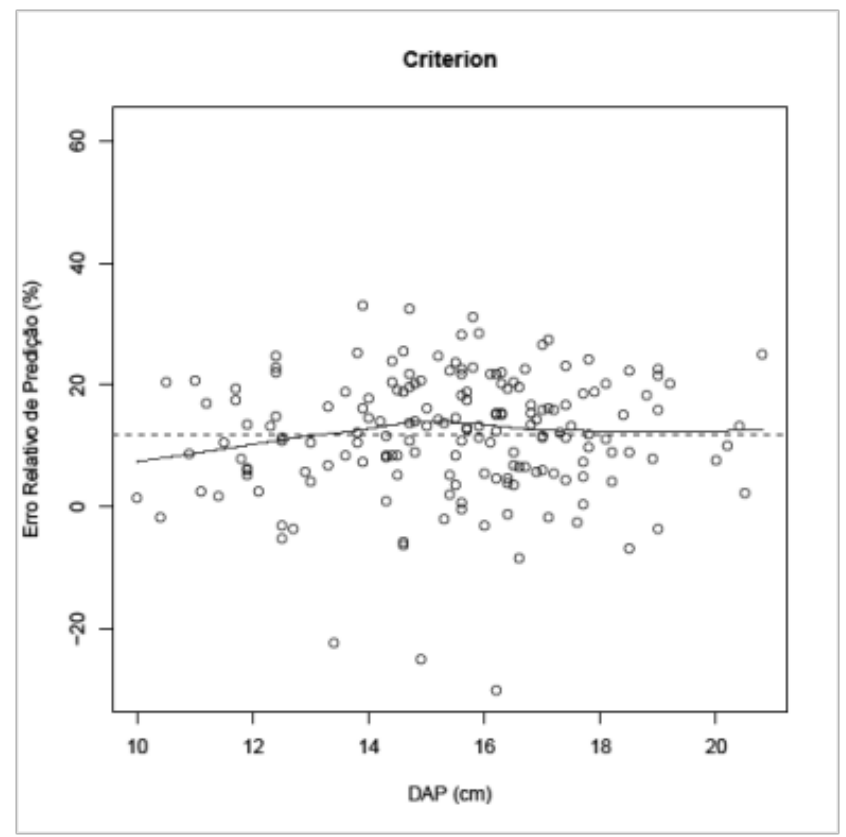

Figura 2. (A) Erro relativo de predição (\%) da cubagem pelo Criterion de forma não destrutiva em função do DAP (cm) e (B) com os dados do Criterion para o ajuste através do melhor modelo ajustado em função do DAP $(\mathrm{cm})$.

A

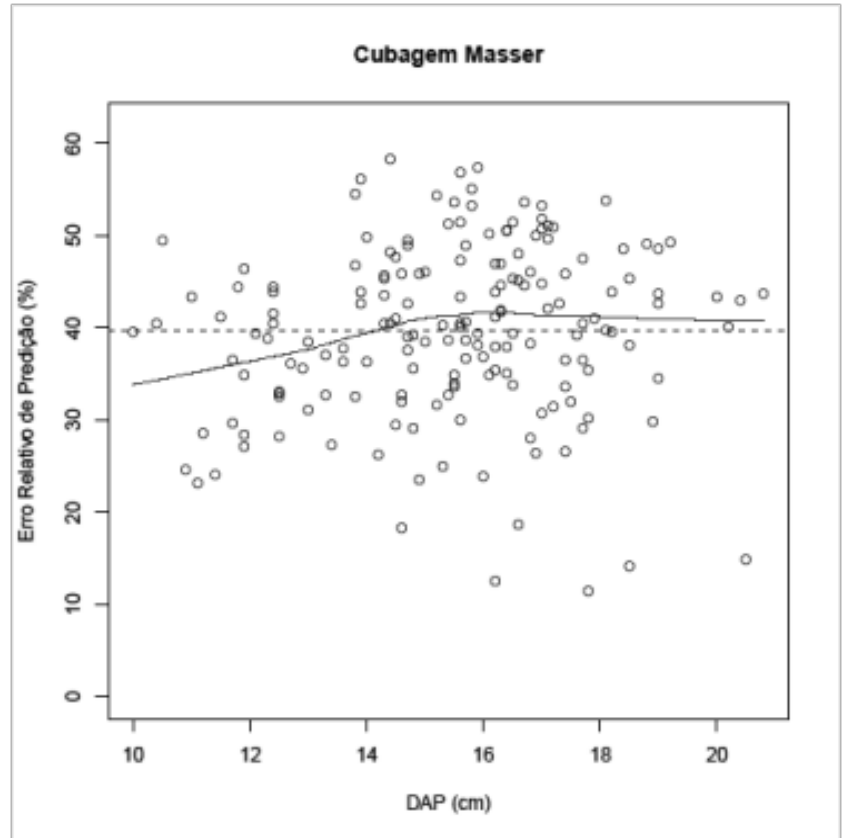

B

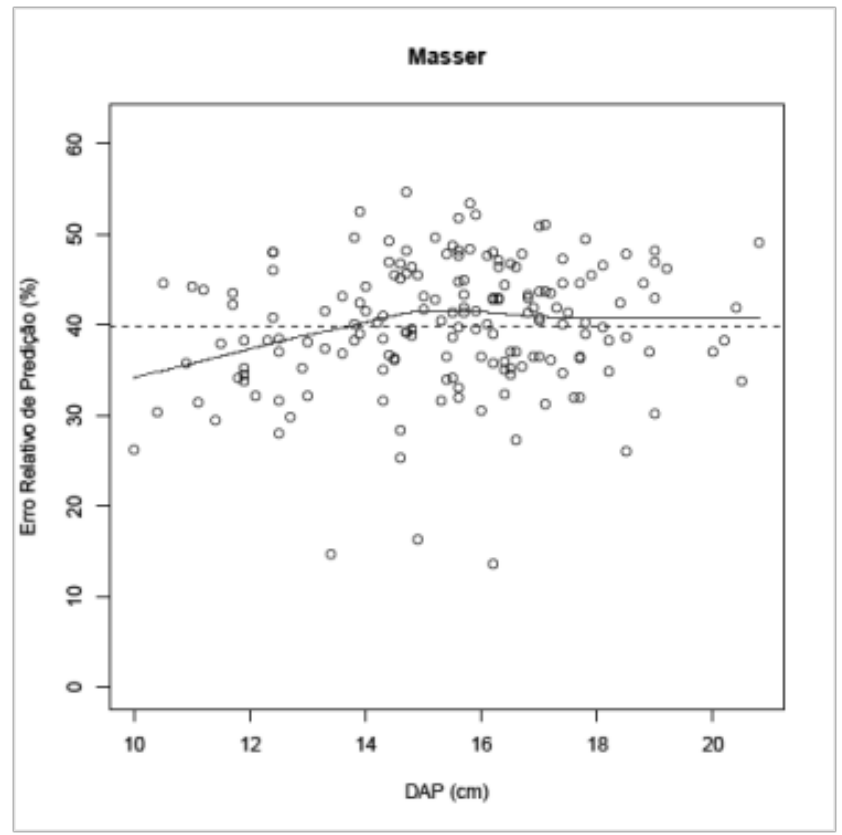

Figura 3. (A) Erro relativo de predição (\%) da cubagem pelo RC3H da Masser de forma não destrutiva em função do DAP $(\mathrm{cm})$ e (B) com os dados do RC3H da Masser para o ajuste através do melhor modelo ajustado em função do DAP $(\mathrm{cm})$. 


\section{Conclusão}

Os resultados deste estudo mostraram que pode haver grandes diferenças nas estimativas de biomassa do fuste para os métodos de determinação não destrutiva, quando comparadas aos valores da cubagem rigorosa. Assim, analisando a acurácia dos dois dendrômetros ópticos para a predição da biomassa do fuste concluiu-se que, em ambos os casos, a variável de interesse foi subestimada. Ainda assim, o Criterion foi o instrumento que forneceu as estimativas mais exatas.

De modo geral, houve um bom ajuste para todos os métodos testados. Através da distribuição gráfica do erro de predição percebeu-se um comportamento mais uniforme dos resíduos para os valores obtidos do melhor modelo do que aqueles da técnica volumétrica, exceto para os dados da cubagem rigorosa tradicional. O erro de predição relativo com a cubagem rigorosa forneceu uma média superestimada de aproximadamente $1 \%$. Com a predição do Criterion foi subestimado em média $11 \%$ e com o RC3H o erro foi subestimado em torno de $40 \%$. Porém, em ambos os casos não houve uma tendência do erro de predição da biomassa, ou seja, em todos os métodos estudados a variância se manteve homogênea. Comparando-se estes resultados com o erro comumente admitido nos inventários florestais - em torno de $10 \%$ nota-se que a utilização do dendrômetro óptico Criterion para predição da biomassa sob forma não destrutiva pode fornecer estimativas próximas do aceitável. Entretanto, determinar a biomassa através desta metodologia exige grandes cuidados na observação das variáveis, tais como, distância árvore-observador, presença de obstáculos e presença de vento nas medições. Assim, quanto mais precisa for a estimativa dos diâmetros medidos sob forma não destrutiva, mais confiável será a estimativa da biomassa estocada nas florestas.

\section{Referências}

BURNHAM, K. P.; ANDERSON, D. R. Model selection and multimodel inference: practical information theoretic approach. 2nd. ed. New York: Springer, 2002. 488 p.

FEARNSIDE, P. M.; LEAL FILHO, N.; FERNANDES, F. M. Rainforest burning and the global carbon budget: biomass, combustion efficiency, and charcoal formation in the Brazilian Amazon. Journal of Geophysical Research, Washington, US, v. 98, n. D9, p. 16733-16743, 1993.

HIGUCHI, N.; SANTOS, J.; RIBEIRO, R. J.; MINETTE, L.; BIOT, Y. Biomassa da parte aérea da vegetação da floresta tropical úmida de terra-firme da Amazônia Brasileira. Acta Amazônica, Manaus, v. 28, n. 2, p. 153-166, 1998.
HIGUCHI, N.; CARVALHO JÚNIOR, J. A. Fitomassa e conteúdo de carbono de espécies arbóreas da Amazônia. In: SEMINÁRIO EMISSÃO X SEQÜESTRO DE CO2: UMA NOVA OPORTUNIDADE DE NEGÓCIOS PARA O BRASIL, 1994, Rio de Janeiro. Anais... Rio de Janeiro: Companhia Vale do Rio Doce, 1994. p. 125-145.

LIU, C. J.; HUANG, X.; EICHEMBERGER, J. K. Using laser technology for measuring trees. In: IUFRO conference, 1993, Morgantown, WV. Modern methods of estimating tree and log volume. Morgantown, W.V.: West Virginia University Publication Services, [1993]. $13 \mathrm{p}$.

MACHADO, S. A.; FIGUEIREDO FILHO, A. Dendrometria. 2. ed. Curitiba, 2006. 309 p.

MACHADO, S. A.; NASCIMENTO, R. G. M.; AUGUSTYNCZIK, A. L. D.; SILVA, L. C. R.; FIGURA, M. A.; PEREIRA, E. M.; TEO, S.J. Comportamento da relação hipsométrica de Araucaria angustifolia no capão da Engenharia Floretal da UFPR. Pesquisa Florestal Brasileira, Colombo, n. 56, p. 5-16, jan./jun. 2008.

MELO, A. C. G.; DURIGAN, G. Fixação de carbono em reflorestamentos de matas ciliares no Vale do Paranapanema, SP, Brasil. Scientia Forestalis, Piracicaba, SP, n. 71, p. 149-154, 2006.

NELSON, B. W.; MESQUITA, R.; PEREIRA, J.; SOUZA, S. G.; BATISTA, G.; COUTO, L. B. Allometric regressions for improved estimate off secondary forest biomass in the central Amazon. Forest Ecology and Management, Amsterdam, n. 117, p. 149-167, 1999.

NICOLETTI, M. F. Comparação de métodos não-destrutivos de cubagem de árvores em pé visando à determinação da biomassa. 2011. Dissertação (Mestrado em Recursos Florestais) - Escola Superior de Agricultura Luiz de Queiroz, Universidade de São Paulo, Piracicaba, SP.

PANSHIN, A. J.; DE ZEEUW, C. Textbook of wood technology. 4. ed. New York: McGraw-Hill, 1980. 404 p.

SANQUETTA, C. R. Métodos de determinação de biomassa florestal. In: SANQUETTA, C. R.; WATZLAWICK, L. F.; BALBINOT, R. M.; ZILIOTTO, M. A. B.; GOMES, F. S. (Ed.). As florestas e o carbono. Curitiba: UFPR, 2002. p. 119-140.

SCHNEIDER, P. R. Análise de regressão aplicada à engenharia florestal. 2 ed. Santa Maria, RS: UFSM, CEPEF, 1998.

SILVEIRA, P. Ajuste de modelos matemáticos para estimar biomassa aérea em floresta ombrófila densa. Revista Floresta, Curitiba, v. 39, n. 4, p. 743-752, out./dez. 2009.

SILVA, H. D.; FERREIRA, C. A.; CORREA, R. S.; BELLOTE, A. F. J.; TUSSOLINI, E. L. Alocação de biomassa e ajuste de equações para estimativa de biomassa em compartimentos aéreos de Eucalyptus benthamii. Boletim de Pesquisa Florestal, Colombo, $\mathrm{n}$. 49, p. 83-95. jul./dez. 2004.

SOARES, C. P. B.; LEITE, H. G.; GORGENS, E. B. Equações para estimar o estoque de carbono no fuste de árvores individuais em plantios comerciais de eucalipto. Revista Árvore, Viçosa, MG, v. 29, n. 5, p. 711-718, ago. 2005.

WILLIAMS, M. S.; CORMIER, K. L.; BRIGGS, R. G.; MARTINEZ, D. R. Evaluation of the Barr \& Stroud FP15 and criterion 400 laser dendrometers for measuring upper stem diameters and heights. Forest Science, Washington, DC, v. 45, n. 1, p. 53-61. 1999. 
